\title{
DIE GRUNDPROBLEME DER EVANGELISATION HEUTE NACH KAROL KARD. WOJTYEA Eine theologische Untersuchung
}

Ein gewiß unerschöpfliches Thema. Der Untertitel beschränkt es auf den theologischen Gesichtspunkt. Aber auch hier mußte eine Auswahl von Problemen erfolgen, die wie jede andere Auswahl unter einem bestimmten Aspekt getroffen werden mußte und daher auch diskutabel ist. Ich möchte aber, daß dieser Gesichtspunkt nicht nur der von Karol Wojtyła bleibt, sondern zu dem wird, der für die Evangelisation gerade Europas von wesentlichster Bedeutung ist. Ihnen, meine Damen und Herren, möchte ich die Beurteilung überlassen, ob dieser Gesichtspunkt von mir zutreffend gewählt und ausgedrückt wurde.

Im Zusammenhang damit muß - meiner Meinung nach — zunächst das betrachtet werden, was im Titel als „heute" bezeichnet wurde, um dann weiter eine vertiefende Analyse durchführen zu können im Hinblick auf das gegenseitige Verhältnis der vertikalen und horizontalen Interpretierung des Christentums. Diese Probleme bedingen meines Erachtens wesentlich die Auffassung der Evangelisation in Europa. Eine Betonung dieser Fragen soll uns nicht nur die Realien der Evangelisationsarbeit der Kirche zeigen, sondern auch deren großen Wert für den gegenwärtigen Menschen, das heißt, daß die Evangelisation ihm wirklich das Evangelium bringt — also die „gute Botschaft”.

* In der vorliegenden Form wurde dieser Vortrag an der Fakultät für katholische Theologie der Ruhr-Universität Bochum am 10. November 1987 gehalten. Ein Jahr vorher, im November 1986, hatte der Autor zu diesem Thema auch an den Universitäten in Regensburg und Passau gesprochen. Die hier vorgetragenen Überlegungen wurden grundgelegt von Kardinal Karol Wojtyła während der Bischofssynode 1974 in Rom zu dem Thema: „Über die Evangelisation in der Welt von heute". Der Autor hatte als dessen Privattheologe an dieser Synode teilgenommen und bei der Ausarbeitung der grundlegenden Gedanken mitgearbeitet. Inzwischen hat Papst Johannes Paul II. am 7.12.1991 die Enzyklika Redemptoris missio über die fortdauernde Gültigkeit des missionarischen Auftrages veröffentlicht. Ende desselben Jahres fand in Rom die Sondersynode für Europa statt. Die Erklärung dieser Synode „Seien wir Zeugen Christi, der uns befreit hat” vom 13.12.1991 hat das Prinzip der Neuevangelisierung Europas formuliert. Es scheint dem Autor, daß der Inhalt seines Vortrages von 1987 weiterhin aktuell ist. 
Wenn von Evangelisation „heute” gesprochen wird, dann denke ich an die Bezeichnung des Zweiten Vatikanunms: „... in der Welt von heute”. Wie bekannt, ist das ein Fragment des Titels der pastoralen Konstitution über die Kirche „Gaudium et spes”. Für die Evangelisation als theologisches Thema hat der Begriff „Welt” nach K. Wojtyła eine enorme Bedeutung.

Den in dieser Konstitution „Gaudium et spes” steht dem Lehramt der Kirche vor Augen ,die Welt der Menschen, das heißt die ganze Menschheitsfamilie mit der Gesamtheit der Wirklichkeiten, in denen sie lebt; die Welt, der Schauplatz der Geschichte der Menschheit, von ihren Unternehmungen, Niederlagen und Siegen geprägt; die Welt, die nach dem Glauben der Christen durch die Liebe des Schöpfers begründet ist und erhalten wird; die unter die Knechtschaft der Sünde geraten, von Christus aber, dem Gekreuzigen und Auferstandenen, durch Brechung der Herrschaft des Bösen befreit wurde; bestimmt, umgestaltet zu werden nach Gottes Heilsratschluß und zur Vollendung zu kommen" (GS 2).

Übereinstimmend mit dieser Auffassung des Begriffs „Welt”, müssen wir mit K. Wojtyła zunächst feststellen, daß die Welt mitgestaltet, was wir als Situation des Subjekts der Evangelisation verstechen, also der Kirche, die „in der Welt von heute" evangelisiert. Die Welt, so verstanden, als „Schauplatz der Geschichte der Menschheit", wird in eine bestimmte Zeitspanne eingerahmt, deren Zeichen sorgfältig gelesen werden müssen — in Übereinstimmung mit der Lehre der Offenbarung und des Magisteriums.

Der Begriff „Welt” im Sinne des Konzils kann ebenso nach K. Wojtyła das Objekt der Evangelisation betreffen, da die Kirche sich nicht nur mit der Evangelisation „in der Welt”, sondern auch — und das vor allem - mit der Evangelisation der Welt selbst befaßt. Das ist ein besonders wichtiges Problem, denn es weist auf das Verhältnis zwischen der Evangelisation und dem Evangelium selbst und der Welt hin. Die Verkündigung des Evangeliums ist nichts anderes als eine Erklärung des Geheimnisses der Welt und des Menschen im Licht des Heilsplanes Gottes, denn die Welt ist schließlich im angeführten Text der Pastoralkonstitution die Heimat der Menschheitsfamilie. Von einer solchen Auffassung der Welt sagt das Zweite Vatikanum deutlich, daß die Welt durch die Liebe des Schöpfers begründet ist, erhalten wird und von Christus erlöst wurde. Die so verstandene Welt wird zum Objekt der Evangelisation und bildet gleichzeitig ihre besondere Begründung.

Die Aufnahme des Evangeliums ist also nichts anderes als die Erfüllung des Rechtes und das gleichzeitige Nachkommen der grundsätzlichen Pflichten des gesamten Geschöpfes vor Gott. „Denn dem Herrn gehört die Erde und was sie erfüllt" (1 Kor. 10,26; vgl. Ps. 24[23],1). Alle, die das Evangelium in der Welt 
verkünden, sollen sich dessen bewußt und davon überzeugt sein. Die Verkündigung des Auferstandenen sollte mit dem Zweiten Vatikanum auf dem Grund des Glaubens stehen, daß nur er „dem Menschen selbst den Menschen offenbart hat” und stets offenbart. „Tatsächlich klärt sich nur im Geheimnis des fleischgewordenen Wortes das Geheimnis des Menschen wahrhaft auf" (GS 22). Daher bedeutet auch die Evangelisation nichts anderes als die Erflüllung des Rechtes der ganzen in der Welt lebenden Menschheitsfamilie und ein gleichzeitiges Nachkommen der Pflichten angesichts des Erlösers. Darin liegt die moralische Kraft sämtlicher Evangelisationstätigkeit, und das Fundament dieser Kraft ist in der obigen Auffassung der Welt und des Menschen zu suchen.

Wenn die Kirche die Welt evangelisiert und gleichzeitig in ihr das Werk des Evangeliums erfüllt, dann schöpft sie ihre große Liebe zur Welt aus den offenbarten Weisheiten, die sie verkündet. „Denn Gott hat die Welt so sehr geliebt, das er seinen einzigen Sohn hingab" (Joh. 3,16). Über die Welt, die der Schöpfer am Anfang den Menschen übergab, ist gesagt worden: unterwerft sie euch! (vgl. Gen. 1,28). Im Gedanken und Herzen der evangelisierenden Kirche darf die Welt, in der der Mensch lebt und sich im Namen des menschlichen Fortschrittes entwickelt, nicht getrennt werden von der Welt, deren Geheimnis das Evangelium Jesu Christi erklärt.

Es gibt aber noch einen dritten Begriff der Welt der Meinung von K. Wojtyła nach in den oben angeführten Worten des Konzils - ein Begriff, der bei der Besprechung der Evangelisation der Welt im Europa von heute nicht verschwiegen werden darf. Diesem Begriff möchte ich besondere Aufmerksamkeit widmen, da er - manchmal vollkommen bewußt - aus dem christlichen Bild des Menschen entfernt wird. Gerade die Pastoralkonstitution enthüllt im Text „über die Macht des Bösen” und über die Welt, die „unter die Knechtschaft der Sünde geraten" ist diesen Begriff der Welt, der im offenbarten fleischgewordenen Wort eine ausreichend selbstverständliche Deutung findet. Durch die „Macht des Bösen” wurde die Welt schon im Gedanken und Herzen des ersten Menschen, des Mannes und der Frau, der Absicht und dem heilbringenden Auftrag des Schöpfers entgegengesetzt als die, die vollkommen das Bedürfnis der menschlichen Natur befriedigen kann. Diese Versuchung, die sich immer wieder erneuert, kehrt in unserem Zeitalter, in dem wir sehen, wie der Fleiß des Menschen die umgebende Welt gestaltet, mit neuer Gewalt zurück. Einige Richtungen des heutigen „Säkularismus”, zu denen verschiedene Tendenzen der menschlichen Wissenschaft und Tätigkeit führen, entfalten sich in Form des praktischen und theoretischen Materialismus.

Diese Versuchung ist mit der Form des Anthropozentrismus verbunden, der den Menschen an die Stelle Gottes stellt — wie bei den ersten Eltern: „ihr werdet sein wie Gott" (Gen. 3,5) - und proklamiert die vollkommene Unabhängigkeit des Menschen von seinem Schöpfer und Erlöser. Viele der heutigen Vertreter des gegenwärtigen Existentialismus begründen das mit einer bewun- 
dernswerten Meisterschaft. Zugleich muß hier beigefügt werden, daß in diesen Formen eines „Anti-Evangeliums” vieles aus dem Evangelium verborgen ist — jedoch im entgegengesetzten Sinne.

Wenn das Werk der Evangelisation in der gegenwärtigen Welt betrachtet wird, kann also die Tatsache nicht vergessen werden, daß es in dieser Welt verschiedene Formen eines Anti-Evangeliums und verschiedene Formen einer Anti-Evangelisation gibt. Es scheint, daß in diesen Formen die Begriffe Welt und Mensch den Kern und den Grund des Gegensatzes zum Evangelium Jesu Christi bilden. Deshalb ist die Evangelisation der Welt zu jeder Zeit - zweifellos in unserer - mit einem Kampf werbunden, zu dem der Herr seine Apostel ganz offen vorbereitet hat: „Ihr aber, macht euch darauf gefaßt: Man wird euch um meinetwillen vor die Gerichte bringen, in den Synagogen mishandeln und vor Statthalter und Könige stellen, damit ihr vor ihnen Zeugnis ablegt. Vor dem Ende aber muß allen Völkern das Evangelium verkündet werden. Und wenn man euch abführt und vor Gericht stellt, dann macht euch nicht im voraus Sorgen, was ihr sagen sollt; sondern was euch in jener Stunde eingegeben wird, das sagt! Denn nicht ihr werdet dann reden, sondern der Heilige Geist” (Mk. 13,9-11). Jesus Christus wird in jeder Zeit „ein Zeichen sein, dem widersprochen wird", nach den Worten Simeons in Lk. 2,34. Das gleiche betrifft sein Evangelium. Paulus war sich dessen bewußt, als er an die Epheser schrieb: „Denn wir haben nicht gegen Menschen aus Fleisch und Blut zu kämpfen, sondern gegen die Fürsten und Gewalten, gegen die Beherrscher dieser finsteren Welt, gegen die bösen Geister des himmlischen Bereichs" $(6,12)$. Mit diesen Worten hat er deutlich gemacht, daß der mit der Evangelisation verbundene Kampf vor allem einen geistigen Charakter hat, auch wenn Elemente dieser Welt in ihm eine wesentliche Rolle spielen.

Wenn wir die Evangelisation in der Welt von heute betrachten, dann müssen wir feststellen, daß sie heute wohl mehr als früher unter Umständen einer starken Opposition verläuft, sowohl auf intellektuellem als auch auf ideologischem Gebiet sowie auch auf der Ebene der Moralität und der gesamten menschlichen Existenz. In einigen Teilen der Welt - ganz besonders in Europa - wächst die Zahl der Menschen, die sich zu einer Anti-Evangelisation bekennt, gewaltig an, so daß der „Widerspruch” öffentlich wirkt und das Evangelium Jesu Christi wirklich als ein „Zeichen des Widerspruchs” erscheint.

Es darf nicht vergessen werden, daß Christus und seine Apostel so auch lehren. Es soll jedoch stets als Grundstein der Evangelisation der Glaube an die Welt und an den Menschen gelegt werden, denn dieser Glaube führt trotz der vorhandenen Sünde und der Macht des Bösen in der Welt — zum Glaubensbekenntnis, daß viel bedeutender das Licht und die Kraft des Vaters sind, die sich in der Macht des Erlösers konzentrieren und uns den Weg des Heiles im Heiligen Geiste weisen. 
Genau genommen handelt es sich hier um die Vorstellung der Prioritäten der Evangelisation, die unaufgebbar sind, auch wenn sie früher und heute in der theologischen Tradition unterschiedlich lebendig bleiben. Es handelt sich dabei nach K. Wojtyła um das ewige Heil (salus aeterna) und um die Förderung des Menschen (promotio humana). Eine Evangelisation, die diese Fragen nicht ernstnehmen sollte, müßte wohl als nicht vollkommen christlich bezeichnet werden. Sie sind eng miteinander verbunden und gegenseitig bedingt. Die Gegenüberstellung irgendeiner Absolutierung des einen oder des anderen hat weder der Evangelisation noch der Kirche gut getan.

\section{Salus aeterna}

Der christliche Glaube hat immer, erklärt K. Wojtyła, den apostolischen Dienst der Kirche mit dem ewigen Heil verbunden. Paulus schämte sich nicht des Evangeliums, denn: „Es ist eine Kraft Gottes, die jeden rettet, der glaubt, zuerst den Juden, aber ebenso den Griechen. Denn im Evangelium wird die Kraft Gottes offenbart aus Glauben zum Glauben, wie es in der Schrift heißt: Der aus Glauben Gerechte wird leben" (Röm. 1,16-17). Also ist der Glaube der evangelisierenden Kirche innerlich mit der Hoffnung auf das ewige Leben verbunden, „das der wahrhaftige Gott schon vor ewigen Zeiten verheißen hat" (Tit. 1,2). In demselben Sinn empfiehlt Petrus in seinem ersten Brief den „Auserwählten, die als Fremde [...] in der Zerstreuung leben", in den Gebieten von Klein-Asien, daß sie mit Freude die Prüfungen des Glaubens tragen. „Gepriesen sei der Gott und Vater unseres Herrn Jesus Christus: Er hat uns in seinem großen Erbarmen neu geboren, damit wir durch die Auferstehung Jesu Christi von den Toten eine lebendige Hoffnung haben und das unzerstörbare, makellose und unvergängliche Erbe empfangen, das im Himmel für euch aufbewahrt ist. Gottes Macht behütet euch durch den Glauben, damit ihr das Heil erlangt, das am Ende der Zeit offenbart werden soll. Deshalb seid ihr voll Freude, obwohl ihr jetzt vielleicht kurze Zeit unter mancherlei Prüfungen leiden müßt. Dadurch soll sich euer Glaube bewähren, und es wird sich zeigen, daß er wertvoller ist als Gold, das im Feuer geprüft wurde und doch vergänglich ist. So wird (eurem Glauben) Lob, Herrlichkeit und Ehre zuteil bei der Offenbarung Jesu Christi. Ihn habt ihr nicht gesehen, und dennoch liebt ihr ihn, ihr seht ihn auch jetzt nicht, aber ihr glaubt an ihn und jubelt in unsagbarer, von himmlischer Herrlichkeit verklärter Freude, da ihr das Ziel des Glaubens erreichen werdet, euer Heil" (1 P. 1,3-9).

In dieser Lehre vereint sich die „Prüfung des Glaubens” mit der lebendigen Erwartung des „unzerstörbaren Erbes”, zu dem wir erneut geboren wurden 
vom himmlichen Vater in seiner großen Barmherzigkeit durch die Auferweckung Christi. Das ewige Heil ist das Ziel des Glaubens und dadurch auch das Ziel der Evangelisation; es ist zugleich eine reiche Gabe unseres Vaters im Himmel, wie es die Worte von Johannes bestätigen: „Gott hat uns das ewige Leben gegeben und dieses ewige Leben ist in seinem Sohn" (1 Joh. 5,11). Daher schreibt Paulus an Timoteus über sein Leiden: „Das alles erdulde ich um der Auserwählten willen, damit auch sie das Heil in Christus Jesus und die ewige Herrlichkeit erlangen" und zeigt gleichzeitig auf seine eigene Weise den Weg unseres Heiles in Christus: „Wenn wir mit Christus gestorben sind, werden wir auch mit ihm leben; wenn wir standhaft bleiben, werden wir auch mit ihm herrschen, wenn wir ihn verleugnen, wird auch er uns verleugnen. Wenn wir untreu sind, bleibt er doch treu, denn er kann sich selbst nicht verleugnen" (2 Tim. 2,10-13).

Die Apostel, die durch das Licht aus der Höhe und die Kraft des Heiligen Geistes zu Zeugen Jesu Christi wurden, verkündigten auf diese Weise das ewige Heil und bereiteten den Menschen den Weg zum Heil durch den Glauben. Auch die Kirche, die die gegenwärtige Welt evangelisiert, bekennt ähnlich ihren eschatologischen Charakter, was besonders deutlich wird im 7. Kapitel der dogmatischen Konstitution über die Kirche Lumen gentium unter der überschrift: „Der endzeitliche Charakter der pilgernden Kirche und ihre Einheit mit der himmlichen Kirche". Es gibt nichts, was angemessener wäre für das Evangelium und was wesentlicher wäre für das Werk der Evangelisation als das Zeugnis vom ewigen Heil, zu dem der Mensch in seinem ganzen irdischen Leben von seinem Schöpfer geführt wird. „Die Kirche weiß sehr wohl”, heißt es in der Konstitution Gaudium et spes, „daß Gott, dem sie dient, allein die Antwort ist auf das tiefste Sehnen des menschlichen Herzens, das an den Gaben der Erde nie voll sich sättigen kann" (GS 41).

\section{Promotio humana}

Die Ausrichtung auf das ewige Heil ist in der heutigen Evangelisationsarbeit nach $\mathrm{K}$. Wojtyła eng verbunden mit ihren Bemühungen um die richtige Förderung des Menschen, die die Kirche immer - zumindest wenn es um die Prinzipien geht - als integralen Teil ihrer Mission und Tätigkeit auf Erden betrachtete. Davon sprechen viele Zeugnisse und Unternehmungen im Laufe der gesamten bisherigen Geschichte des Christentums. Die Lehre des Zweiten Vatikanums spricht viel über dieses Thema, insebesondere, wenn es seine Aufmerksamkeit dem Problem der Würde der menschlichen Person zuwendet, deren Sicherung wesentlich die vielseitige und integrale Entwicklung der Welt konstituiere. In der Welt, so heißt es in der Pastoralkonstitution, „wächst auch 
das Bewußtsein der erhabenen Würde, die der menschlichen Person zukommt, da sie die ganze Dingwelt überragt und Träger allgemeingültiger sowie unverletzlicher Rechten und Pflichten ist" (GS 26). Der Entwicklung der Welt hilft „der Geist Gottes, dessen wunderbare Vorsehung den Lauf der Zeiten leitet und das Antlitz der Erde erneuert... Der Sauerteig des Evangeliums hat im Herzen des Menschen einen unbezwingbaren Anspruch auf Würde erweckt und erweckt ihn auch weiter" (GS 26). Die richtige Förderung des Menschen war also durch Gott den Vater und Schöpfer selbst vorgesehen und hat ihre volle Bestätigung in seinem Sohn und Erlöser gefunden. Sie wird unter den Menschen weitergeführt durch den Heiligen Geist mit Vermittlung der Kirche, „die zugleich Zeichen und Schutz der Transzendenz der menschlichen Person ist" (GS 76).

Jesus Christus, der einzige „treue Zeuge” (Apg. 3,14) seiner Mission, hat stark betont, daß in der Verküngigung des Evangeliums allen Geschöpfen die Liebe beisteht. Denn „Gott ist die Liebe” (1 Joh. 4,8), wer also auf Erden Zeuge des Wortes Gottes sein will, der muß Liebe haben und mit ihr täglich leben. Das Evangelium des Heiles ist mit dem Gebot der Liebe zu Gott und zu dem Nächsten verbunden (vgl. Lk. 10,27). Paulus schreibt darüber: „Die Liebe ist ausgegossen in unsere Herzen durch den Heiligen Geist” (Röm. 5,5) und weist auf ihren privilegierten Platz unter anderen Gaben und Tugenden hin (vgl. 1 Kor. 13,1-13). Sie ist der Schlüssel zum Erlangen des ewigen Heiles und zugleich der Entwicklung des Menschen, die, wie es die Pastoralkonstitution lehrt, darauf beruht, ,daß der Wert des Menschen mehr in ihm selbst liegt als in seinem Besitz" (GS 35). Es ist also kein Wunder, daß die Kirche in ihrem Werk der Evangelisation der heutigen Welt auf verschiedene Weise zum Tun der Liebe aufruft. Die Verkünder des Evangeliums dürfen nie den ethischen Wert des Gebotes der Liebe vergessen, werder bei der Erziehung der menschlichen Person noch im gemeinsamen Leben der Gesellschaft. Gerade die ethische Kraft der Liebe, hoch geschätzt auch von Ungläubigen, soll durch die Evangelisaton alle Lebensbereiche innerlich durchdringen und, die Würde des Menschen achtend, zur Entwicklung des Menschen beitragen.

In der heutigen Welt, die stark technokratisiert ist, deren Struktur oft auf materialistischen Grundlagen beruht, in der gesagt wird, daß die menschliche Person das endgültige Ziel sei, sie praktisch aber zu anderen Zwecken als Mittel und Werkzeug ausgenutzt wird - kann die hier besprochene Promotion des Menschen in Wirklichkeit nur durch die Vermittlung des Wortes des Evangeliums erfolgen, seiner Macht und Tat, verbunden mit dem Versprechen, das einem geduldigen Suchen des ewigen Heiles gegeben ist: „Euch aber muß es zuerst um sein Reich und seine Gerechtigkeit gehen, dann wird euch alles andere dazugegeben" (Mt. 6,33). Die Fragen des ewigen Heiles und der Promotion des Menschen dürfen also nicht voneinander getrennt werden, weder in der gesellschaftlichen Tätigkeit, noch im Leben der einzelnen Menschen, da diese 
beiden Gebiete durch die Schöpfung wie die Erlösung eng miteinander verbunden sind. Daher dürfen die vertikale und die horizontale Dimension der Evangelisation nicht zu sehr durch verschiedene evangelisierende Unternehmungen in Gegensatz zueinander gebracht werden. Die Menschen haben kein Recht, diese Prioritäten der Tätigkeit der ganzen Kirche zu ändern, die doch von Christus bestimmt wurden, indem er, unter anderem, sprach: „Getrennt von mir könnt ihr nichts vollbringen" (Joh. 15,5).

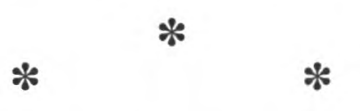

Es ist selbstverständlich, daß die Theologie der Evangelisation insgesamt sehr viele unterschiedliche Probleme umfaßt, die alle ebenfalls eines vertieften Nachdenkens bedürften. Außerdem kommen die Probleme auf verschiedenen Kontinenten oder in bestimmten Gegenden nicht immer in gleicher Weise zum Vorschein. In Lateinamerika wird die Evangelisation durch die Befreiungstheologien dominiert. Die afrikanische Theologie interessiert sich besonders für den Grundsatz der Inkulturation, die Indigenisation genannt wird, also eine Verwurzelung der Verkündigung des Evangeliums in der eigenen Kultur bedeutet. Der Zusammenstoß des Christentums mit den nicht christlichen Religionen Asiens rückt ihren theologischen Status an die erste Stelle. Schließlich sind die Länder, in denen die atheistische marxistische Ideologie gefördert wurde, einer besonderen Prüfung im religiösen Leben ihrer Bürger ausgesetzt. Da ich mir dieser und anderer Tatsachen, z. B. der Dechristianisierung in allen europäischen und amerikanischen Landern, besonders in den Grosstätten, bewußt bin, habe ich in meinem Vortrag nur einige zentrale Probleme aufgegriffen.

Am Ende möchte ich mit Karol Wojtyła, indem ich mich auf die Bischofssynode von 1974 beziehe, noch auf ein Problem aufmerksam machen. Es ist ein theologisch sehr wichtiges Thema. Ich denke an die Rolle des Heiligen Geistes im Werk der Evangelisation der Welt. In der Überzeugung der Synodenbischöfe ist der Heilige Geist der Hauptverkünder des Evangeliums. Er dringt in die Tiefen Gottes ein und sprach durch die Propheten. Er enthüllt den Menschen das Geheimnis des Heiles. Er macht ihre Herzen bereit, das Wort Gottes zu hören und macht es in ihrem Leben fruchtbar. Allein der Heilige Geist führt die Menschen zum Glauben. Auch ist seine Anwesenheit in den nichtchristlichen Religionen und in einigen Laienorientierungen nicht ganz auszuschließen.

Wenn diese theologische Doktrin in der Vergangenheit, auch auf dem Zweiten Vatikanischen Konzil, bekannt war und gelehrt wurde, so erfährt heute die Kirche quasi „experimentell” die Wirkung des Heiligen Geistes in den charismatischen Bewegungen. Daher kam die Forderung des Primats der Leh- 
re über den Heiligen Geist in der Theologie der Evangelisation. Heute kann nämlich nur der mutig Christus verkündigen, der sich vom Heiligen Geist führen läßt, der in ihm seine ganze Hoffnung sieht und nicht an dem Erfolg der Evangelisátion zweifelt, auch dort, wo keine sichtbaren Ergebnisse zu erblicken sind. Das ist zweifellos ein gewisses „Novum”, das nach dem Vatikanum II durch die „Orthopraxie” in die Pneumatologie der Evangelisation eingebracht wurde. 